\title{
The Search for Supernova-Produced Radionuclides in Terrestrial Deep-Sea Archives
}

\author{
J. Feige ${ }^{\mathrm{A}, \mathrm{G}}$, A. Wallner $^{\mathrm{A}, \mathrm{B}, \mathrm{C}}, S$. R. Winkler $^{\mathrm{A}}, S$. Merchel $^{\mathrm{D}}$, L. K. Fifield $^{\mathrm{B}}$, \\ G. Korschinek ${ }^{\mathrm{E}}$, G. Rugel ${ }^{\mathrm{D}}$, and D. Breitschwerdt ${ }^{\mathrm{F}}$ \\ A University of Vienna, Faculty of Physics - Isotope Research, VERA Laboratory, \\ Währinger Straße 17, 1090 Vienna, Austria \\ ${ }^{B}$ Department of Nuclear Physics, The Australian National University, Canberra, \\ ACT 0200, Australia \\ ${ }^{\text {C } A N S T O, ~ L o c k e d ~ B a g ~ 2001, ~ K i r r a w e e, ~ D C, ~ N S W ~ 2232, ~ A u s t r a l i a ~}$ \\ ${ }^{\mathrm{D}}$ HZDR, Bautzner Landstraße 400, 01328 Dresden, Germany \\ ${ }^{\mathrm{E}}$ Physics Department, TU Munich, James-Franck-Str., 85748 Garching, Germany \\ F Department of Astronomy and Astrophysics, TU Berlin, Germany, \\ Hardenbergstrasse 36, 10623 Berlin, Germany \\ ${ }^{\mathrm{G}}$ Corresponding author. Email: jenny.feige@univie.ac.at
}

\begin{abstract}
An enhanced concentration of ${ }^{60} \mathrm{Fe}$ was found in a deep ocean crust in 2004 in a layer corresponding to an age of $\sim 2 \mathrm{Myr}$. The confirmation of this signal in terrestrial archives as supernovainduced and the detection of other supernova-produced radionuclides is of great interest. We have identified two suitable marine sediment cores from the South Australian Basin and estimated the intensity of a possible signal of the supernova-produced radionuclides ${ }^{26} \mathrm{Al},{ }^{53} \mathrm{Mn},{ }^{60} \mathrm{Fe}$, and the pure $r$-process element ${ }^{244} \mathrm{Pu}$ in these cores. The finding of these radionuclides in a sediment core might allow us to improve the time resolution of the signal and thus to link the signal to a supernova event in the solar vicinity $\sim 2 \mathrm{Myr}$ ago. Furthermore, it gives us an insight into nucleosynthesis scenarios in massive stars, condensation into dust grains and transport mechanisms from the supernova shell into the solar system.
\end{abstract}

Keywords: Nuclear reactions, nucleosynthesis, abundances — ISM: bubbles — ISM: supernova remnants

Received 2011 December 1, accepted 2012 February 16, published online 2012 March 21

\section{Introduction}

Stars with masses larger than $8 \mathrm{M}_{\odot}$ end their life in a supernova (SN) explosion. The nuclides that were synthesized in the late phases of the star and during the explosion are ejected and entrained in the supernova shell, which expands rapidly into the surrounding interstellar medium. If such an event happens in the solar vicinity, the remnant may leave certain traces in terrestrial archives. Ejecta of a SN contain freshly produced long-lived radionuclides; eventually there might be a chance of finding such radionuclides in terrestrial archives.

Such a signal of possible SN origin has been detected by Knie et al. (2004) in the hydrogenetic ferromanganese crust $237 \mathrm{KD}$, which was recovered from the equatorial Pacific. The radionuclide ${ }^{60} \mathrm{Fe}$ with $t_{1 / 2}=(2.62 \pm 0.04)$ Myr (Rugel et al. 2009) was measured with accelerator mass spectrometry (AMS) utilizing the MP-tandem accelerator in Munich. The signature was detected again in the same crust sample in layers at a depth of $6-8 \mathrm{~mm}$ in a second measurement in the same laboratory, using different chemical separation schemes for AMS target preparation (Fitoussi et al. 2008).
The age of the deep ocean's crust was determined through ${ }^{10} \mathrm{Be}$ dating. Based on the half-life of $t_{1 / 2}\left({ }^{10} \mathrm{Be}\right)=(1.51 \pm 0.06) \mathrm{Myr}$ (Hofmann et al. 1987), Knie et al. (2004) estimated a time range of 2.4-3.2 Myr for the layer that contained the anomaly. Fitoussi et al. (2008) measured the ${ }^{10} \mathrm{Be}$ content consistently and re-evaluated this result using $t_{1 / 2}\left({ }^{10} \mathrm{Be}\right)=(1.36 \pm 0.07)$ Myr (Nishiizumi et al. 2007). The half-life of ${ }^{10} \mathrm{Be}$ was recently remeasured by Chmeleff et al. (2010) and Korschinek et al. (2010) and the suggested value is now $(1.387 \pm 0.012)$ Myr. Following Fitoussi et al. (2008), we calculate the time profile with this new value and obtain a time range of 1.74-2.61 Myr. Figure 1 shows the recalculated age profile of the sediment core, including the data of Knie et al. (2004) and Fitoussi et al. (2008).

A hint as to recent $\mathrm{SN}$ activity in the solar vicinity is the existence of a thin hot cavity in the local interstellar medium (ISM), the Local Bubble (LB), which embeds our solar system. Its extensions are about $200 \mathrm{pc}$ in the Galactic plane and $600 \mathrm{pc}$ perpendicular to it. Superbubbles are formed by several SN explosions, creating blast waves that push away the surrounding medium. 


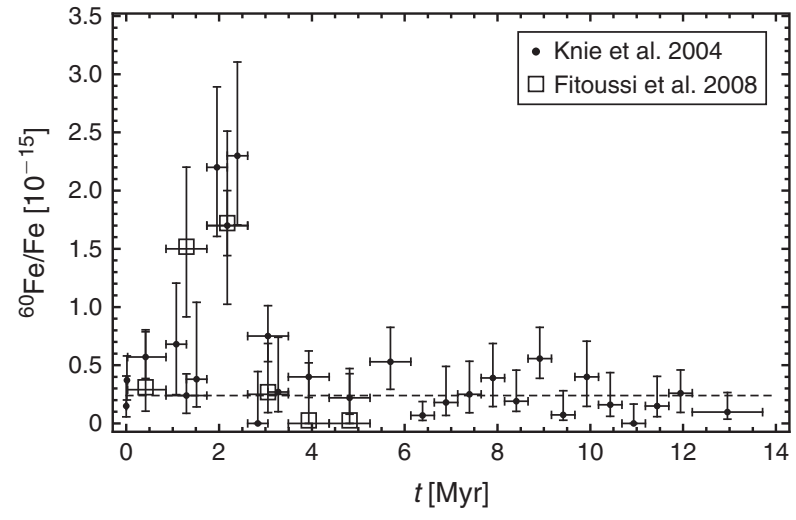

Figure 1 Measured ${ }^{60} \mathrm{Fe} / \mathrm{Fe}$ ratios versus the age of the layer calculated with $t_{1 / 2}\left({ }^{10} \mathrm{Be}\right)=1.387 \mathrm{Myr}$. The black circles show the measurements by Knie et al. (2004), the white squares the data of Fitoussi et al. (2008)

They leave thin hot gas inside, encircled by a shell consisting mainly of accumulated material but also of SN ejecta. The latest age calculations of the LB, based on comparison between observations and simulations of ion column density ratios, suggest a value of 13.6-13.9 Myr (Avillez \& Breitschwerdt 2012). Fuchs et al. (2006) identified a stellar moving group as having crossed the solar neighborhood and determined about 14-20 SN explosions to form the LB, the last of these occurring approximately $0.5 \mathrm{Myr}$ ago. The remaining stars belong today to the subgroups UCL (Upper Centaurus Lupus) and LCC (Lower Centaurus Crux) of the Sco-Cen association.

It is suggested that the stars that created the LB also contributed to the ${ }^{60} \mathrm{Fe}$ excess on Earth (Berghöfer \& Breitschwerdt 2002). Neuhäuser et al. (2011) have searched for the birthplace of neutron stars and their runaway companions to corroborate this theory.

To obtain further evidence for recent $\mathrm{SN}$ explosions in the solar vicinity, we have identified two suitable marine sediments from the Indian Ocean, the Eltanin Piston Cores ELT 45-21 and ELT 49-53. Both sediment cores originate from a location south-west of Australia, $38^{\circ} 58.7^{\prime} \mathrm{S}$, $103^{\circ} 43.1^{\prime} \mathrm{E}$ and $37^{\circ} 51.6^{\prime} \mathrm{S}, 100^{\circ} 01.7^{\prime} \mathrm{E}$ (Allison \& Ledbetter 1982). They were recovered from depths of 4237 and $4248 \mathrm{~m}$ in the years 1970 and 1971 . With accumulation rates of 3-4 mm kyr ${ }^{-1}$, the cores grow much faster than the ferromanganese crust $\left(\sim 2 \mathrm{~mm} \mathrm{Myr}^{-1}\right)$. Such a high resolution allows a more precise dating of SN signals compared with previous measurements and thus one may constrain the time period of the incoming shock wave. In contrast to a crust, the formation process of a sediment is well understood. The uptake efficiency, the amount of an element to be incorporated into the sediment, might be up to $100 \%$; in a manganese crust this value is only vaguely determined. If the SN signal is detectable in these two sediments, it allows us to draw conclusions regarding the production rate of the measured radionuclides and transport mechanisms from the SN envelope to Earth. The long-lived radionuclides ${ }^{26} \mathrm{Al},{ }^{53} \mathrm{Mn},{ }^{60} \mathrm{Fe}$, with half-lives of the order of the $\mathrm{SN}$ event $\sim 2 \mathrm{Myr}$ ago, and the pure $r$-process element ${ }^{244} \mathrm{Pu}$ are particularly applicable in this case.

\section{Summary of Previous Measurements}

More than 15 years ago it was suggested by Ellis et al. (1996) and Korschinek et al. (1996) that there might be a chance of finding long-lived radionuclides in terrestrial archives that were originally produced during a supernova - a possible site for the $r$-process. The first successful measurement was carried out by Knie et al. (1999) in a ferromanganese crust from Mona Pihoa in the South Pacific. An enhanced ${ }^{60} \mathrm{Fe}$ concentration was found in two layers corresponding to a total time range of 0-5.9 Myr, which was later confirmed with improved time resolution in the crust $237 \mathrm{KD}$ by Knie et al. (2004) and Fitoussi et al. (2008). This signal was again observed in another crust (29DR-32) recovered from the North Pacific ocean (G. Korschinek, in preparation).

A measurement of ${ }^{60} \mathrm{Fe}$ of a marine sediment by Fitoussi et al. (2008) gave less promising results. The core taken from the North Atlantic ocean did not show a clear signal. Fitoussi et al. (2008) suggested an overestimation of the fluence in the crust coming from the uncertainty of the uptake factor. Samples from the Moon have been analyzed by Cook et al. (2009) in terms of ${ }^{26} \mathrm{Al},{ }^{60} \mathrm{Fe}$, and ${ }^{10} \mathrm{Be}$. They state that one sample shows a signal that cannot be explained by the production of ${ }^{60} \mathrm{Fe}$ by solar or Galactic cosmic rays. However, the detected signal was one order of magnitude lower than expected.

The presence of another isotope, the $r$-process radionuclide ${ }^{244} \mathrm{Pu}$, was investigated at TU Munich in ferromanganese nodules (Wallner et al. 2000) and in a small piece of the same ferromanganese crust as was used for the ${ }^{60} \mathrm{Fe}$ measurements (Wallner et al. 2004). Independently, Paul et al. $(2001,2003,2007)$ analyzed a marine sediment for possible $r$-process signatures. All these measurements did not result in significant evidence of $\mathrm{SN}$-produced ${ }^{244} \mathrm{Pu}$ in such terrestrial archives. It is not clear whether only a fraction of SN explosions produce $r$-process ${ }^{244} \mathrm{Pu}$ at all. The condensation and transport of ${ }^{244} \mathrm{Pu}$ within dust particles in the SN shell into the solar system is also not understood sufficiently.

Future AMS measurements of SN-produced ${ }^{60} \mathrm{Fe}$ are planned by Bishop \& Egli (2011). By extracting ${ }^{60} \mathrm{Fe}$ from magnetite microfossils produced by magnetotactic bacteria in deep-sea sediments, the search for a possible SN signal is extended to biogenic archives.

\section{Methods}

The red clay piston cores ELT 49-53 and ELT 45-21 might be suitable for detecting possible SN signatures. They were sampled at great depths in the Indian Ocean to minimize contamination from continental input. The ages of the cores were already roughly determined through magnetostratigraphy and biostratigraphy (Allison \& 
Ledbetter 1982). Our sample of ELT 49-53 reaches from a depth of $120-517 \mathrm{~cm}$ and that of ELT $45-21$ from $398-697 \mathrm{~cm}$ below the surface level of the ocean, covering the time range 1.7-3.1 Myr, which includes the time range of the ${ }^{60} \mathrm{Fe}$ peak identified in the ferromanganese crust $237 \mathrm{KD}$. Dating with ${ }^{10} \mathrm{Be}$ gives further details on the age profile. The cores are split into sections of $1 \mathrm{~cm}$ length, corresponding to $\sim 3 \mathrm{kyr}$, with gaps of $8 \mathrm{~cm}$ in between, making 71 samples in total.

Adequate chemical methods to extract $\mathrm{Be}, \mathrm{Al}, \mathrm{Mn}, \mathrm{Fe}$, and $\mathrm{Pu}$ material (each element separately) from the sediment samples are described in Bourlès et al. (1989) and Merchel \& Herpers (1999).

The most sensitive technique to date for detecting long-lived radionuclides is AMS. With this method, the expected small amounts of such radionuclides in terrestrial archives can be quantified.

\section{Estimation of a Possible Signal in the Eltanin Cores \\ $4.1^{10} \mathrm{Be}$}

This isotope is mainly produced by spallation of oxygen and nitrogen by cosmic rays in the Earth's atmosphere. By assuming the cosmic-ray flux was constant over the past few million years, it can be used to date the sections of the core with (Fitoussi et al. 2008)

$$
T_{d}=\frac{t_{1 / 2}\left({ }^{10} \mathrm{Be}\right)}{\ln 2} \ln \left(\frac{C_{0}}{C_{d}}\right) \text {, }
$$

where $T_{d}$ is the age of the layer with depth $d, C_{0}$ the concentration of ${ }^{10} \mathrm{Be}$ at the surface or any reference layer with known age and $C_{d}$ the concentration at depth $d$. An increase of ${ }^{10} \mathrm{Be}$ by an enhanced cosmic-ray flux from a $\mathrm{SN}$ would be small compared with the cosmogenic production. The yield of the ejected ${ }^{10} \mathrm{Be}$ mass in a $\mathrm{SN}$ explosion is at least a factor of $10^{3}$ lower than ${ }^{60} \mathrm{Fe}$ (Woosley \& Weaver 1995) and thus the SN input $2 \mathrm{Myr}$ ago is estimated to be a factor of $10^{3}-10^{4}$ lower than the cosmogenic fraction in a piece of sediment of $1 \mathrm{~cm}$ thickness. Therefore, quantification of any direct ${ }^{10} \mathrm{Be}$ in the sediment cores above terrestrial cosmogenic ${ }^{10} \mathrm{Be}$ is improbable.

\section{$4.2{ }^{26} \mathrm{Al}$ and ${ }^{60} \mathrm{Fe}$}

The radionuclide ${ }^{26} \mathrm{Al}$ is dominantly produced by the ${ }^{25} \mathrm{Mg}(p, \gamma){ }^{26} \mathrm{Al}$ reaction in the core H-burning phase of main sequence stars. A contribution in the later stages of massive stars occurs during $\mathrm{C}$ and $\mathrm{Ne}$ shell burning and explosive $\mathrm{Ne}$ burning. Its half-life is $t_{1 / 2}=(0.717 \pm$ $0.017) \mathrm{Myr}$, which is the weighted mean with standard deviation of the values reported in Samworth et al. (1972), Middleton et al. (1983), Norris et al. (1983), and Thomas et al. (1984), as stated in Auer et al. (2009). ${ }^{60} \mathrm{Fe}$ is synthesized mainly by neutron capture on ${ }^{59} \mathrm{Fe}$ in $\mathrm{He}$ and $\mathrm{C}$ shell burning and during the explosion, when the blast
Table 1. Estimated ${ }^{26} \mathrm{Al}$ fluence $\boldsymbol{F}_{26}\left(\right.$ atoms cm $\left.^{-2}\right)$ in ELT 45-21 and ELT 49-53 with varying uptake factor $U$ and ${ }^{60} \mathrm{Fe} /{ }^{26} \mathrm{Al}$ flux ratio

\begin{tabular}{|c|c|c|c|c|}
\hline \multirow{2}{*}{\multicolumn{2}{|c|}{$\frac{{ }^{60} \mathrm{Fe}}{{ }^{26} \mathrm{Al}}$}} & \multicolumn{3}{|c|}{$U$ [\%] } \\
\hline & & 0.6 & 50 & 100 \\
\hline 0.11 & & $2.7 \times 10^{8}$ & $3.2 \times 10^{6}$ & $1.6 \times 10^{6}$ \\
\hline 0.5 & $F_{26}$ & $5.8 \times 10^{7}$ & $7.0 \times 10^{5}$ & $3.5 \times 10^{5}$ \\
\hline 1 & & $2.9 \times 10^{7}$ & $3.5 \times 10^{5}$ & $1.8 \times 10^{5}$ \\
\hline
\end{tabular}

Table 2. Estimated ${ }^{60} \mathrm{Fe}$ fluence $F_{60}\left(\right.$ atoms $\left.\mathrm{cm}^{-2}\right)$ in ELT 45-21 and ELT 49-53 with varying uptake factor $U$

\begin{tabular}{lccc}
\hline$U[\%]$ & 0.6 & 50 & 100 \\
\hline$F_{60}$ & $1.4 \times 10^{8}$ & $1.6 \times 10^{6}$ & $8.2 \times 10^{5}$ \\
\hline
\end{tabular}

wave moves through the star, roughly in the same region in which ${ }^{26} \mathrm{Al}$ is produced (Limongi \& Chieff 2006).

The ${ }^{26} \mathrm{Al}$ and ${ }^{60} \mathrm{Fe}$ signals in a deep-sea sediment core can be estimated by scaling from the ${ }^{60} \mathrm{Fe}$ fluence estimation in the ferromanganese crust. The average ratio measured in the crust by Knie et al. (2004) was ${ }^{60} \mathrm{Fe} /$ $\mathrm{Fe}=1.9 \times 10^{-15}$ in layers at a depth of $6-8 \mathrm{~mm}$. This corresponds to a ${ }^{60} \mathrm{Fe}$ fluence of $8.2 \times 10^{5}$ atoms cm $\mathrm{cm}^{-2}$ after background correction, which was an averaged ${ }^{60} \mathrm{Fe} / \mathrm{Fe}$ ratio of $2.4 \times 10^{-16}$. Two parameters remain uncertain. One is the uptake factor $U$, as discussed above, the other is the ratio of ${ }^{60} \mathrm{Fe} /{ }^{26} \mathrm{Al}$ as it enters the solar system in the supernova remnant (SNR). Knie et al. (2004) calculated an uptake efficiency of $0.6 \%$. However, a much higher value of $50-100 \%$ is suggested (G. Korschinek, in preparation). The ${ }^{60} \mathrm{Fe} /{ }^{26} \mathrm{Al}$ flux ratio varies from $0.11-0.17$ in observations from the Reuven Ramaty High Energy Solar Spectroscopic Imager (RHESSI) and SPectrometer for INTEGRAL (SPI) and from $0.16-1$ in theoretical models (Limongi \& Chieff 2006). With

$$
\left(\frac{{ }^{60} \mathrm{Fe}}{{ }^{26} \mathrm{Al}}\right)_{m}=\frac{{ }^{60} \mathrm{Fe}}{{ }^{26} \mathrm{Al}} U \times e^{-2.2 \ln 2\left(\frac{1}{2.62}-\frac{1}{0.717}\right)}
$$

the ${ }^{26} \mathrm{Al}$ fluence is calculated, where $\left({ }^{60} \mathrm{Fe} /{ }^{26} \mathrm{Al}\right)_{m}$ is the ratio of the fluences one will measure today. Table 1 shows the ${ }^{26} \mathrm{Al}$ fluence values as expected in the sediments for different parameters of $U$ and the ${ }^{60} \mathrm{Fe} /{ }^{26} \mathrm{Al}$ flux ratio, and Table 2 the corresponding fluence values of ${ }^{60} \mathrm{Fe}$.

The number of atoms per $\mathrm{cm}^{2}$ in the sediment cores is calculated with

$$
F_{s}=\frac{w N_{\mathrm{A}} \rho h}{A}
$$

where $w$ is the mass fraction, $A$ the mass number of the isotope, and $N_{\mathrm{A}}$ Avogadro's constant. The density of the cores was estimated to be $\rho=1.5 \mathrm{~g} \mathrm{~cm}^{-3}$ and the thickness of the layer is $h=1 \mathrm{~cm}$. Such a layer contains a time 
Table 3. Estimated ${ }^{53} \mathrm{Mn}$ fluence $F_{53}\left(\right.$ atoms $\mathrm{cm}^{-2}$ ) in ELT 45-21 and ELT 49-53 with varying uptake factor $U$ and ${ }^{60} \mathrm{Fe} /{ }^{53} \mathrm{Mn}$ flux ratio

\begin{tabular}{|c|c|c|c|c|}
\hline \multirow{2}{*}{$\frac{\overline{{ }^{60} \mathrm{Fe}}}{5{ }^{53} \mathrm{Mn}}$} & & \multicolumn{3}{|c|}{$U[\%]$} \\
\hline & & 0.6 & 50 & 100 \\
\hline $\begin{array}{l}0.1 \\
1\end{array}$ & $F_{53}$ & $\begin{array}{l}1.6 \times 10^{9} \\
1.6 \times 10^{8}\end{array}$ & $\begin{array}{l}2.0 \times 10^{7} \\
2.0 \times 10^{6}\end{array}$ & $\begin{array}{l}9.8 \times 10^{6} \\
9.8 \times 10^{5}\end{array}$ \\
\hline
\end{tabular}

interval of $\sim 3 \mathrm{kyr}$. We assume the sediments contain $\sim 10 \mathrm{wt} \%$ of stable $\mathrm{Al}$ and $\sim 5 \mathrm{wt} \%$ of stable $\mathrm{Fe}$, thus with equation (3) we obtain $3.3 \times 10^{21}$ atoms $\mathrm{cm}^{-2} \mathrm{Al}$ and $8.1 \times 10^{20}$ atoms cm ${ }^{-2}$ of Fe. Therefore, we expect the ${ }^{60} \mathrm{Fe} / \mathrm{Fe}$ ratio of the signal to be in the range $10^{-15}-10^{-13}$ and the ${ }^{26} \mathrm{Al} / \mathrm{Al}$ ratio between $5 \times 10^{-17}$ and $10^{-13}$. The detection limit for measuring ${ }^{26} \mathrm{Al} / \mathrm{Al}$ and ${ }^{60} \mathrm{Fe} / \mathrm{Fe}$ with AMS is $\sim 10^{-16}$, making the most pessimistic values hard to measure. By applying a dedicated chemical sample preparation technique, it is possible to reduce the fraction of stable $\mathrm{Al}$ and achieve an isotope ratio 3-10 times higher (Fitoussi \& Raisbeck 2007).

In contrast to ${ }^{60} \mathrm{Fe},{ }^{26} \mathrm{Al}$ has a much higher natural background. It is produced either by spallation from cosmic rays on Ar in the Earth's atmosphere or in situ in the sediment. The atmospheric production can on the one hand be obtained by scaling the ${ }^{10} \mathrm{Be}$ concentration in the ferromanganese crust. Using an initial atmospheric ratio of ${ }^{26} \mathrm{Al} /{ }^{10} \mathrm{Be}=1.89 \times 10^{-3}$ (Auer et al. 2009), the background is estimated to be $\sim 5 \times 10^{3}$ atoms cm $\mathrm{cm}^{-2}$ in a section of the core of $1 \mathrm{~cm}$ height. Since the uptake of ${ }^{10} \mathrm{Be}$ is also not well known, this can be considered as a lower limit. On the other hand we use the atmospheric production rate of ${ }^{10} \mathrm{Be}$ computed by Masarik \& Beer (2009) of 0.0209 atoms cm $\mathrm{cm}^{-2} \mathrm{~s}^{-1}$ and derive $5 \times 10^{5}$ atoms $\mathrm{cm}^{-2}$ as an upper limit. A source of in situ produced ${ }^{26} \mathrm{Al}$ is the ${ }^{23} \mathrm{Na}(\alpha, n){ }^{26} \mathrm{Al}$ reaction. The $\alpha$-particles are generated in the natural decay chains of ${ }^{232} \mathrm{Th},{ }^{235} \mathrm{U}$, and ${ }^{238} \mathrm{U}$. If the $\mathrm{Na}, \mathrm{U}$, and Th content in the cores is similar to that in the ferromanganese crust, then the production will be in the order of $10^{4}$ atoms $\mathrm{cm}^{-2}$, which is an order of magnitude lower than the estimated upper limit of the atmospheric production. The contribution from micrometeorites can be neglected, as was pointed out by Fitoussi \& Raisbeck (2007).

$4.3^{53} \mathrm{Mn}$

The yield of ${ }^{53} \mathrm{Mn}$ ejected by a SN explosion is similar to or up to an order of magnitude higher than that of ${ }^{60} \mathrm{Fe}$ (Woosley \& Weaver 1995; Rauscher et al. 2002) in the mass range of $11-25 \mathrm{M}_{\odot}$ stars (if we neglect the $13-\mathrm{M}_{\odot}$ star from Woosley \& Weaver 1995). If we take equation (2), replace ${ }^{26} \mathrm{Al}$ with ${ }^{53} \mathrm{Mn}$ and apply the half-life of ${ }^{53} \mathrm{Mn}$ with $t_{1 / 2}=(3.7 \pm 0.4)$ Myr (Honda \& Imamura 1971), we obtain the results shown in Table 3.

${ }^{53} \mathrm{Mn}$ is also produced in cosmic dust by solar cosmic rays. An extraterrestrial flux was calculated by Auer (2008) with $\sim 200$ atoms $\mathrm{cm}^{-2} \mathrm{yr}^{-1}$ of ${ }^{53} \mathrm{Mn}$, which sums up to a fluence of $6 \times 10^{5}$ atoms $\mathrm{cm}^{-2}$ in $1 \mathrm{~cm}$ of our sediment cores.

$4.4{ }^{244} \mathrm{Pu}$

The long-lived radionuclide ${ }^{244} \mathrm{Pu}\left(t_{1 / 2}=(81.1 \pm 0.3)\right.$ Myr, calculated from the $\alpha$ decay half-life of $t_{1 / 2}=$ $(81.2 \pm 0.3)$ Myr (Aggarwal 2006) and spontaneous fission: $t_{1 / 2}=(66 \pm 2)$ Gyr (Holden \& Hoffman 2000) by Lachner et al. (2012)) is a product of the pure $r$-process in massive stars during a $\mathrm{SN}$ explosion. The yield in core collapse supernovae is estimated to be of the order of $M_{\mathrm{ej}}=10^{-8} \mathrm{M}_{\odot}$ for ${ }^{244} \mathrm{Pu}$ (Lingenfelter et al. 2003). The number of atoms per $\mathrm{cm}^{2}$ that reach the Earth is now calculated by spreading the ejected mass over a spherical shell with radius $r$, which is the distance to Earth (Fields et al. 2005):

$$
F=\frac{1}{4} \frac{M_{\mathrm{ej}}}{4 \pi A m_{u} r^{2}}
$$

with $A$ the atomic mass and $m_{u}$ the atomic mass unit. Choosing a very rough estimation for the distance of a SN explosion of $r=100 \mathrm{pc}$, we expect $F_{244} \approx 10^{4} \mathrm{~cm}^{-2}$. This value is small compared with the expected ${ }^{60} \mathrm{Fe}$ fluence, but with the most sensitive AMS facilities for measuring ${ }^{244} \mathrm{Pu}$ it might be possible to detect a SN signal.

If ${ }^{244} \mathrm{Pu}$ is found in the sediment cores, the question remains as to whether it is directly deposited from a single supernova. With its long half-life, ${ }^{244} \mathrm{Pu}$ should be abundant in the ISM through the contribution of multiple nucleosynthesis events. The expanding SN shell accumulates the surrounding medium, which is the low-density medium of the Local Bubble. The hot temperature of the LB of $\sim 10^{6} \mathrm{~K}$ (Berghöfer \& Breitschwerdt 2002) prohibits the formation of molecules and dust grains. Therefore, it is still an open question as to how ${ }^{244} \mathrm{Pu}$ is collected from the ISM and whether it is condensed into dust grains to be deposited on Earth.

\subsection{Deposition Time}

The deposition time of SN ejecta on Earth is calculated using the thin-shell approximation. If we assume that the entire mass is accumulated in the shell, then its thickness $d$ can be estimated with $d / r \sim 1 / 12$ for a strong shock into a monoatomic gas (e.g. Clarke \& Carswell 2007). With a distance $r$ of the SN explosion of the order of $100 \mathrm{pc}$, the shell has a thickness of $\sim 8 \mathrm{pc}$. The SNR expands freely into the ambient medium until the ejected mass is comparable to the swept-up mass of the ISM; subsequently the velocity can be calculated with the Sedov-Taylor solution (e.g. Clarke \& Carswell 2007):

$$
\dot{r}=\frac{2}{5}\left(\frac{2 E}{\rho}\right)^{1 / 2} r^{-3 / 2} .
$$

The energy of a SN explosion is typically $E=10^{51} \mathrm{erg}$ and $\rho$ is the mass density of the LB plasma. This results in a 
value of $5 \times 10^{7} \mathrm{~cm} \mathrm{~s}^{-1}$ at $100 \mathrm{pc}$, which is still a Mach-3 shock. Since the medium inside the LB has a low particle density of $n=5 \times 10^{-3} \mathrm{~cm}^{-3}$ and a temperature of $T=10^{6} \mathrm{~K}$ (Berghöfer \& Breitschwerdt 2002), the pressure is non-negligible. The solution only holds if the kinetic energy of the remnant exceeds the thermal energy of the medium. With pressure $P=2 n k T$ in an ionized gas, where $k$ is the Boltzmann constant, and the adiabatic index $\gamma=5 / 3$ (e.g. Clarke \& Carswell 2007),

$$
\frac{P}{\rho(\gamma-1)}<\frac{1}{2} \dot{r}^{2}
$$

Therefore, we obtain for the deposition time $t_{d}=d / \dot{r} \approx 15 \mathrm{kyr}$. The thin-shell approximation refers to the ISM, which is compressed into a shell by the expanding shock wave. The SN ejecta is accumulated behind this shell, but because $M_{\mathrm{ej}} \ll M_{\mathrm{sw}}$, where $M_{\mathrm{ej}}$ is the ejected mass and $M_{\mathrm{sw}}$ the accumulated ISM mass, we expect the time at which the SN ejecta overruns the solar system to be much lower than $15 \mathrm{kyr}$. It is not clear how the dust grains are transported into the solar system and finally deposited on Earth. These mechanisms might lead to the broadening of a possible signal. Fields et al. (2005) suggest a deposition time of the order of $10 \mathrm{kyr}$.

\section{Conclusion}

We have obtained two sediment cores from the Indian ocean and estimated a possible signal from the SNproduced radionuclides ${ }^{26} \mathrm{Al},{ }^{53} \mathrm{Mn},{ }^{60} \mathrm{Fe}$, and ${ }^{244} \mathrm{Pu}$. In addition, the long-lived radionuclide ${ }^{10} \mathrm{Be}$ is used to date the sediments. The calculations show that it is possible to measure ${ }^{26} \mathrm{Al},{ }^{53} \mathrm{Mn},{ }^{60} \mathrm{Fe}$, and ${ }^{244} \mathrm{Pu}$ with accelerator mass spectrometry, but the cosmogenic background of ${ }^{26} \mathrm{Al}$ and ${ }^{53} \mathrm{Mn}$ might be challenging to distinguish clearly from a SN signal. Because the sediment cores are split into sections of $1 \mathrm{~cm}$ length, which corresponds to a time range of $\sim 3 \mathrm{kyr}$ with gaps of $8 \mathrm{~cm}$ in between, a chance is given that the signal could be located in one of those gaps, corresponding to $\sim 24 \mathrm{kyr}$. The combination of two independent sediments lowers this probability.

We will continue the search for SN-produced radionuclides in terrestrial archives by analyzing the two deepsea sediment cores with respect to the aforementioned $\mathrm{SN}$-produced radionuclides. AMS measurements are planned at the Vienna Environmental Research Accelerator (VERA) facility (University of Vienna), Australian National University (ANU) Canberra, Australia, Technische Universität München, Germany, HelmholtzZentrum Dresden-Rossendorf(HZDR) Dresden, Germany, and Eidgenössische Technische Hochschule (ETH) Zürich, Switzerland.

${ }^{26} \mathrm{Al}$ measurements with high time resolution will be performed; with a half-life of $0.717 \mathrm{Myr}$ it is, like ${ }^{60} \mathrm{Fe}$, sensitive to single $\mathrm{SN}$ events. A positive signal will allow comparison of its intensity with the observed ${ }^{60} \mathrm{Fe}$ signal in the ferromanganese crust (Knie et al. 2004). It provides a check of late stages in massive stars and a comparison with space-borne observations of $\gamma$-ray intensities in our Galaxy (see e.g. Diehl et al. 2010).

In parallel to ${ }^{26} \mathrm{Al}$, the $\mathrm{SN}$-produced radionuclides ${ }^{10} \mathrm{Be},{ }^{53} \mathrm{Mn}$, and ${ }^{60} \mathrm{Fe}$ will be measured in the same sections. ${ }^{10} \mathrm{Be}$ is not sufficiently produced and ejected in a SN explosion, but it is constantly produced in the Earth's atmosphere and can be used to date the sections of the sediment cores.

The search for pure $r$-process ${ }^{244} \mathrm{Pu}$ will be continued with new developments that improve the overall efficiency in AMS measurements, which was the prime limitation on the significance of the previous ${ }^{244} \mathrm{Pu}$ data. While still only upper limits can be given for SN-produced ${ }^{244} \mathrm{Pu}$ on Earth, the goal would be an unambiguous detection of extraterrestrial ${ }^{244} \mathrm{Pu}$, i.e. direct evidence that $r$-processes indeed happened within the last $\sim 100 \mathrm{Myr}$ and can be identified. The ${ }^{244} \mathrm{Pu}$ signal was calculated for a $\mathrm{SN}$ occurring at a distance of $100 \mathrm{pc}$. It is very likely that the ${ }^{60} \mathrm{Fe}$ signal identified by Knie et al. (2004) and Fitoussi et al. (2008) originated from a SN closer than $100 \mathrm{pc}$ to the solar system (Neuhäuser et al. 2011); therefore we might be able to find a higher signal than estimated here.

If all of the above-mentioned elements, which are all ejected in SN explosions, are detected, a clear link to their supernova origin will be provided. The time resolution of the sediment cores is 1000 times higher than for the ferromanganese crust, which will help to narrow the time range of the explosion in the solar vicinity.

It is not yet clear how dust is formed in SN ejecta and how the grains are transported to Earth. The radionuclides are only able to overcome the ram pressure of the solar wind and the interplanetary magnetic field if they are condensed into dust particles (Athanassiadou \& Fields 2011). The dust condensation efficiency in SNRs is a critical value and not very well known yet. It ranges from $10^{-4}$ in observations to 1 in theoretical simulations (Ouellette et al. 2010). Comparing our measurements with theoretical SN models should give an insight into the formation of dust particles in SNRs and nucleosynthesis scenarios in massive stars and during the explosion.

\section{Acknowledgments}

This work is funded by the Austrian Science Fund (FWF), project no. AI00428 and AP20434, through the European Science Foundation Collaborative Research Project CoDustMas. We thank the Antarctic Research Facility, Florida State University, US (C. Sjunneskog) for generously providing the sediment cores and K. Knie (GSI Helmholtzzentrum für Schwerionenforschung $\mathrm{GmbH}$, Darmstadt, Germany) for placing the ${ }^{60} \mathrm{Fe}$ measurement data at our disposal.

\section{References}

Aggarwal, S. K., 2006, RCA, 94, 397

Allison, E. \& Ledbetter, M. T., 1982, MarGe, 46, 131

Athanassiadou, T. \& Fields, B. D., 2011, NewA, 16, 229

Auer, R. M., 2008, PhD Thesis, University of Vienna 
Auer, R. M., Wagenbach, D., Wild, E. M., Wallner, A., Priller, A., Miller, H., Schlosser, C. \& Kutschera, W., 2009, E\&PSL, 287, 453

Avillez, M. A. \& Breitschwerdt, D., 2012, A\&A, 539, L1

Berghöfer, T. W. \& Breitschwerdt, D., 2002, A\&A, 390, 299

Bishop, S. \& Egli, R., 2011, Icar, 212, 960

Bourlès, D., Raisbeck, G. M. \& Yiou, F., 1989, GCA, 53, 443

Chmeleff, J., et al., 2010, NIMPB, 268, 192

Clarke, C. \& Carswell, R., 2007, Principles of Astrophysical Fluid Dynamics (Cambridge: Cambridge University Press)

Cook, D. L., et al., 2009, Lunar and Planetary Institute Science Conference Abstracts, 40, 1129

Diehl, R., et al., 2010, A\&A, 522, A51

Ellis, J., Fields, B. D. \& Schramm, D. N., 1996, ApJ, 470, 1227

Fields, B. D., Hochmuth, K. A. \& Ellis, J., 2005, ApJ, 621, 902

Fitoussi, C. \& Raisbeck, G. M., 2007, NIMPB, 259, 351

Fitoussi, C., et al., 2008, PhRvL, 101, 12

Fuchs, B., Breitschwerdt, D., de Avillez, M. A., Dettbarn, C. \& Flynn, C., 2006, MNRAS, 373, 993

Hofmann, H. J., et al., 1987, NIMPB, 29, 32

Holden, N. E. \& Hoffman, D. C., 2000, PApCh, 72, 8

Honda, M. \& Imamura, M., 1971, PhRvC, 4, 1182

Imamura, M., Inoue, T., Nishiizumi, K. \& Tanaka, S., 1979, ICRC, 2, 304

Knie, K., Korschinek, G., Faestermann, T., Wallner, C., Scholten, J. \& Hillebrandt, W., 1999, PhRvL, 83, 1

Knie, K., Korschinek, G., Faestermann, T., Dorfi, E. A., Rugel, G. \& Wallner, A., 2004, PhRvL, 93, 17

Korschinek, G., Faestermann, T., Knie, K. \& Schmidt, C., 1996, Radiocarbon, 38, 68
Korschinek, G., et al., 2010, NIMPB, 268, 187

Lachner, J., et al., 2012, PhRvC, 85, 1

Limongi, M. \& Chieffi, A., 2006, ApJ, 647, 483

Lingenfelter, R. E., Higdon, J. C., Kratz, K.-L. \& Pfeiffer, B., 2003, ApJ, 591, 228

Masarik, J. \& Beer, J., 2009, JGR, 114, D11103

Merchel, S. \& Herpers, U., 1999, RCA, 84, 215

Middleton, R., Klein, J., Raisbeck, G. \& Yiou, F., 1983, NIMPR, 218,430

Neuhäuser, R., Tetzlaff, N., Eisenbeiss, T. \& Hohle, M. M., 2011, preprint (ArXiv e-prints, 1111.0453)

Nishiizumi, K., Imamura, M., Caffee, M.W., Southon, J. R., Finkel, R. C. \& McAninch, J., 2007, NIMPB, 258, 403

Norris, T. L., Swart, P. K., Wright, I. P., Grady, M. M. \& Pillinger, C. T., 1983, JGRS, 88, B331

Ouellette, N., Desch, S. J. \& Hester, J. J., 2010, ApJ, 711, 597

Paul, M., et al., 2001, ApJ, 558, L133

Paul, M., et al., 2003, NuPhA, 719, C29

Paul, M., et al., 2007, JRNC, 272, 243

Rauscher, T., Heger, A., Hoffman, R. D. \& Woosley, S. E., 2002, ApJ, 576, 323

Rugel, G., et al., 2009, PhRvL, 103, 7

Samworth, E. A. \& Olness, J. W., 1972, PhRvC, 5, 138

Thomas, J. H., Rau, R. L., Skelton, R. T. \& Kavanagh, R. W., 1984, PhRvC, 30, 385

Wallner, C., et al., 2000, NIMPB, 172, 333

Wallner, C., Faestermann, T., Gerstmann, U., Knie, K., Korschinek, G., Lierse, C. \& Rugel, G., 2004, NewAR, 48, 145

Woosley, S. E. \& Weaver, T. A., 1995, ApJS, 101, 181 